\title{
FPGA BASED PORTABLE EMBEDDED REAL TIME HUMIDITY AND TEMPERATURE MEASURING SYSTEM
}

\author{
G Sivakumar', A Arul Paligan², K N Mohan ${ }^{3}$, V Shubha ${ }^{4}$ \\ ${ }^{1}$ Airport Instrumentation, CSIR-NAL, Bangalore, India \\ ${ }^{2}$ Airport Instrumentation, CSIR-NAL, Bangalore, India \\ ${ }^{3}$ Surface Instruments, IMD, Pune, India \\ ${ }^{4}$ Airport Instrumentation, CSIR-NAL, Bangalore, India
}

\begin{abstract}
A high resolution, cost effective, robust portable embedded FPGA based real time humidity and temperature measuring system has been indigenously developed with provision for local and remote data storage. The system not only measures humidity and temperature but also has provision for insitu recording of the data in the system and can be accessed at any place through web enabling. The system is portable with low power consumption capable of operating both on mains AC 230V or using DC battery. Usage of high resolution real time data acquisition with simultaneous sampling decimates required data via secured communication system. The software has been developed for Data acquisition, signal conditioning, processing and publishing in LabVIEW Real Time FPGA platform.
\end{abstract}

Keywords-temperature; humidity; embedded system; labview; dew point temperature; FPGA

\section{INTRODUCTION}

Aviation weather reports give utmost importance to relative humidity as this is one of the most important parameters to calculate the take of / landing distance and angle of climb for an aircraft. Sudden decrease in temperature when atmosphere has high relative humidity is an indication of rapid fall of dew point temperature that can affect the aviation operation. This is due to the water vapour content being high during maximum relative humidity condition. Also high rate of decrease in air temperature accelerates the process of condensation of water vapour on the aircraft surface. Depending on the material used for aircraft surface being metallic or composite, water vapour will either be converted to ice or water on aircraft wing, compressor or carburetor of engine. Formation of ice in the above mentioned aircraft parts can cause serious issues due to increase in weight of the aircraft and/or blockage of nozzles. This results in poor lift, bringing down the efficiency of the aircraft engine and decrease in climb angle of the aircraft.

Management of Air traffic becomes very important as maximum aircraft movement should be planned when the atmosphere has low humidity and moderate temperature. Hence it becomes very important to measure accurately humidity and temperature on a real time basis for planning of efficient aircraft operations. There is also need for developing robust, high resolution integrated temperature and humidity measuring system to monitor these parameters inside the aircraft cabin. In view of the above a rugged, portable high accuracy real time Humidity and Temperature measuring system has been developed using Laboratory Virtual Instrument Electronic Workbench (LabVIEW) Field Programmable Gate Array (FPGA) platform.

\section{METHOD AND IMPLEMENTATION}

An accurate embedded real time humidity and temperature measuring system using integrated Temperature \& Relative Humidity sensor (Elektronik make model EE06-FT1A1) has been developed. The sensor gives Temperature \& Humidity as voltage outputs. These voltages ranging from 0 to $1 \mathrm{~V}$ for both temperature $\left(-40\right.$ to $\left.+60^{\circ} \mathrm{C}\right)$ \& Relative Humidity (0 to $100 \%$ ) will be digitized using two channels of 24-bit simultaneous sampling Analog to Digital (A/D) module of any data acquisition system [1]. We have used National Instruments (NI) embedded real time controller under FPGA platform (compact Reconfigurable Input Output \{cRIO\} 9076) with 9239 A/D module. Software under LabVIEW FPGA has been developed to acquire humidity and temperature data simultaneously at a sampling rate of $1 \mathrm{KS} / \mathrm{s}$ (Kilo Samples/second). Digital filtering techniques with suitable low pass filters to get better Signal to Noise $(\mathrm{S} / \mathrm{N})$ ratio have been deployed. The advantage of data acquisition software developed under LabVIEW FPGA uses the virtual instrumentation concept wherein it is equivalent using 15 standalone instruments [2].

The digitized voltage in FPGA platform is transferred to real time controller using Direct Memory Access (DMA). Proprietary Software has been developed in LabVIEW Real Time platform to convert the digitized humidity and temperature signals into engineering units. Fig.1. gives the photograph of the measuring and monitoring system set up. 


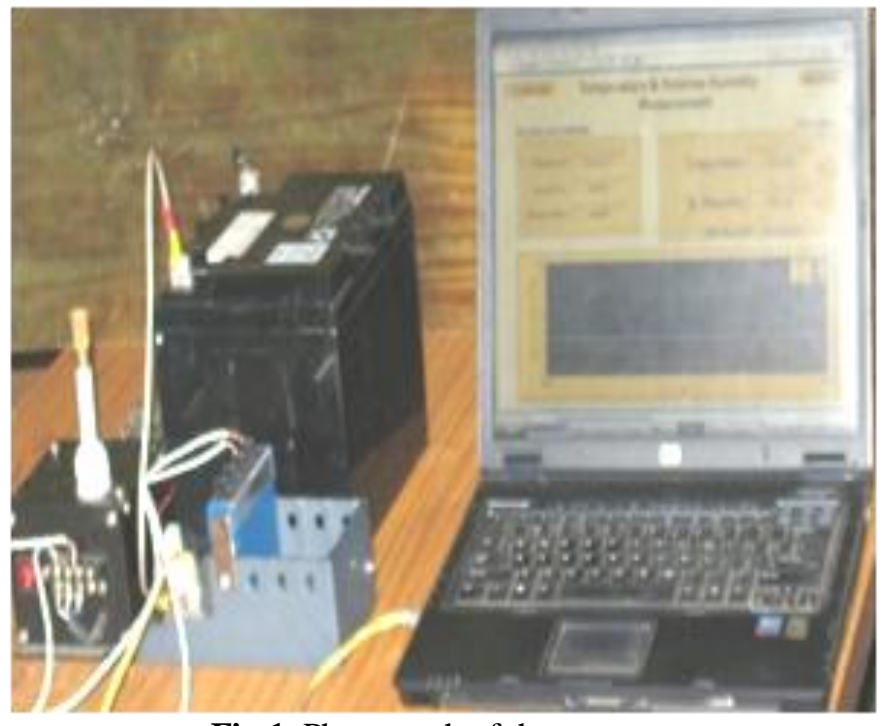

Fig.1. Photograph of the set up.

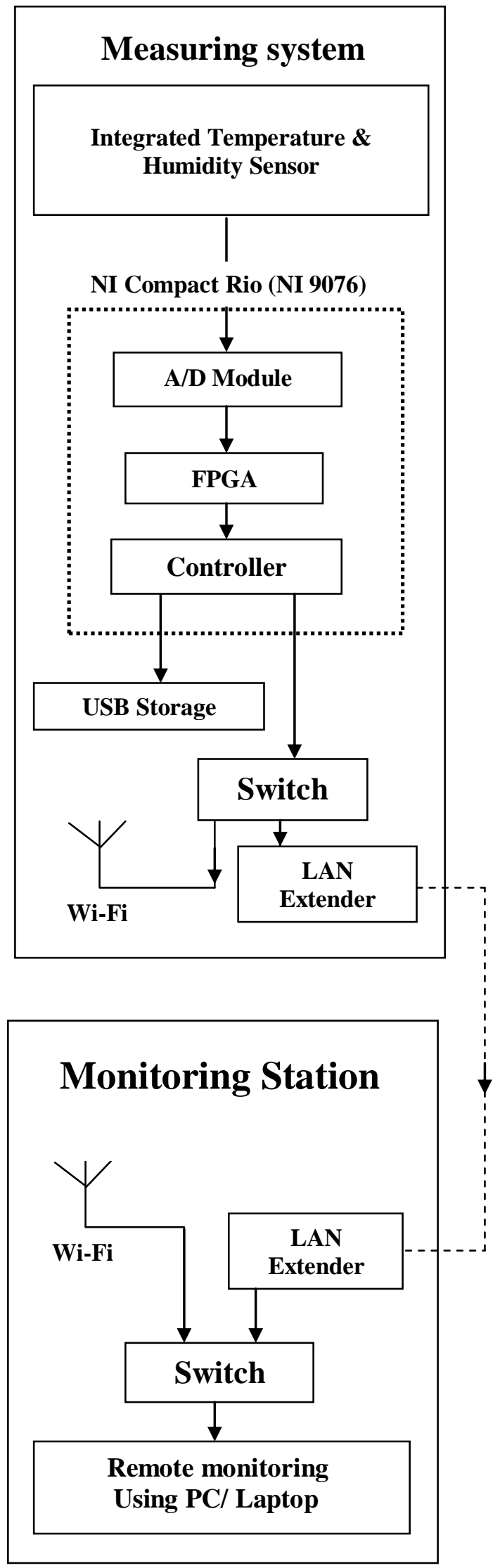

Fig.2. Block diagram of the system

Fig 2 gives the block diagram of the system wherein the complete flow chart of the process is depicted. 


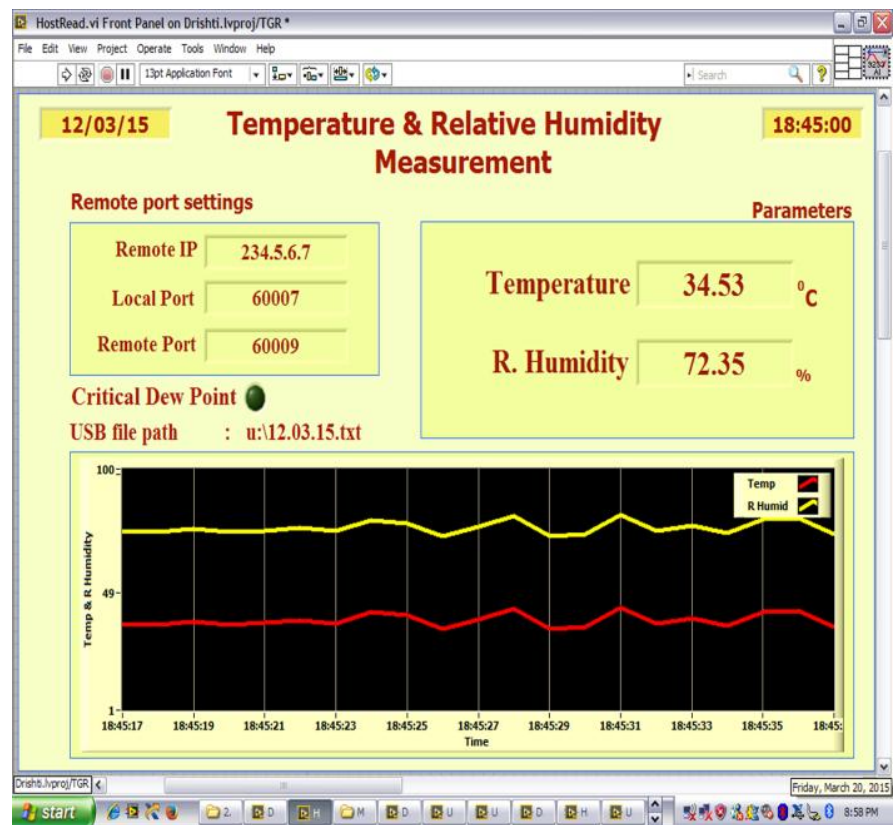

Fig.3. Front panel of the software

The humidity and temperature are displayed in a real time graph with auto scaling facility [3]. There is also continuous display of the actual humidity and temperature. The front panel of the software is given in Fig 3.

The real time data is archived in the external USB memory device with time stamping at every 10 seconds. The file containing 24 hour data of both humidity and temperature is archived as a text file in the USB port with that particular date as the file name for future usage. This is also indicated in the front panel (Fig 3). Fig 4 gives an example of how data file is seen. The front panel of the software /system is written onto Ethernet port for remote accessing and monitoring. The data will be continuously updated every second. The front panel can be viewed in a PC or laptop at the remote location in any web browser viz., Google Chrome, Internet explorer, Mozilla Firefox etc with the IP address of embedded controller.

\begin{tabular}{|c|c|c|c|}
\hline \\
\hline \multicolumn{4}{|c|}{$\begin{array}{l}\text { 12.03.2015 - Notepad } \\
\text { File Edit Format View Help }\end{array}$} \\
\hline Date & \multicolumn{3}{|c|}{ 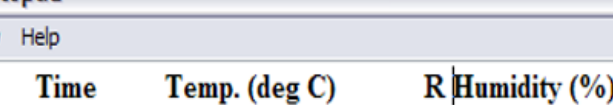 } \\
\hline 12.03 .2015 & $18: 45: 00$ & 34.53 & 72.35 \\
\hline 12.03.2015 & $18: 45: 10$ & 34.60 & 73.36 \\
\hline $12: 03.2015$ & $18: 45: 20$ & 35.00 & 73.00 \\
\hline 12.03.2015 & $18: 45: 30$ & 35.01 & 71.55 \\
\hline 12.03.2015 & $18: 45: 40$ & 34.67 & 72.46 \\
\hline $12: 03.2015$ & $18: 45: 50$ & 36.00 & 72.67 \\
\hline 12.03 .2015 & $18: 46: 00$ & 35.34 & 72.48 \\
\hline 12.03.2015 & $18: 46: 10$ & 34.12 & 73.68 \\
\hline $12: 03.2015$ & $18: 46: 20$ & 32.43 & 75.39 \\
\hline 12.03.2015 & $18: 46: 30$ & 33.43 & 74.28 \\
\hline 12.03 .2015 & $18: 46: 40$ & 32.26 & 73.49 \\
\hline $12: 03.2015$ & $18: 46: 50$ & 33.00 & \\
\hline 12.03 .2015 & $18: 47: 00$ & 34.33 & $\begin{array}{l}72.00 \\
73.24\end{array}$ \\
\hline
\end{tabular}

Fig.4. Example of archived data file
This can cover a distance of only 100 meters. Twisted pair cable with LAN Extenders can transfer the data to a far off location (up to 10kms) like Air traffic Control room from the runway. In some Airports, it will be difficult to have land line cabling. In such cases Wi-Fi communication is a boon. The data with $\mathrm{Wi}-\mathrm{Fi}$ radio modem operating at free band frequencies of 2.4 or $5.8 \mathrm{GHz}$ can be received over a aerial distance of $10 \mathrm{kms}$. Remote accessing beyond a distance of $10 \mathrm{kms}$ can be extended by Wi-Fi repeaters [4].

\section{RESULT AND DISCUSSION}

The Integrated humidity and Temperature sensor along with embedded data acquisition system deployed in airports near runways acts like a standalone field site system. To protect the system from harsh environmental conditions it is housed in a Fiber Reinforced Plastic (FRP) enclosure with provision for air circulation to monitor the actual atmospheric condition. The system has been calibrated in an environmental chamber for various temperature, humidity conditions with rapid Dew point fall. The system met all the conditions required for usage at stringent atmospheric conditions. The LED based annunciator indicates the sharp variation in dew point.

\section{CONCLUSION}

Present paper demonstrates an Embedded Real Time high precision rugged Humidity and Temperature measurement system. This system is very useful for the fast response realtime measurement and monitoring of the relative humidity and temperature in aviation field. It can be used to compare, calibrate and evaluate the performance of the existing fieldsite systems. Also provides a quick temporary substitute incase of breakdown of the system already in operation. Further in future, this system can be very easily extended to integrate many such sensors viz., wind speed, wind direction, pressure etc., situated in different location at runway using single embedded controller.

The developed system is a high precision tool to diagnose atmospheric conditions due to tornados and cyclones. It can also perform as a laboratory equipment for calibration of pressurized climatic chamber. It is especially useful in places where unmanned remote operations are required such as in radioactive regions.

\section{REFERENCES}

[1] Data sheet of humidity sensor EE06-FT1A1.

[2] Nation Instruments, "LabVIEW Reference Manual", USA, 2010.

[3] Moghavvemi M. and Tan. S. "A reliable and economically feasible remote sensing system for temperature and relative humidity measurement". Sensors and Actuators, pp, 181-185, 2005.

[4] M. Benghanem, A Low Cost wireless Data Acquisition System for weather Station, vol. 35, no. 4, pp. 862-872, April 2010. 